\title{
An experimental study of yield stress fluid displacement flows in a moving annulus
}

\author{
Amin Amiri", Roozbeh Mollaabbasi, Faïçal Larachi, Seyed Mohammad Taghavi \\ Chemical engineering department \\ Université Laval \\ Québec, Canada \\ *amin.amiri.1@ulaval.ca
}

\begin{abstract}
In this paper, the displacement flow of a viscoplastic fluid by a non-Newtonian fluid in a vertical annulus is experimentally studied. The two fluids have a very small density difference. The light displaced fluid is placed below the heavy displacing fluid. Buoyancy, the imposed flow velocity and the annulus motion are the important flow parameters. In regard to the annulus motion, two scenarios are considered: first, the annulus is stationary; Second, the annulus slowly reciprocates with an amplitude of $100 \mathrm{~mm}$ and the frequency of $0.1 \mathrm{~Hz}$. Our experimental results demonstrate that the displacement flow efficiency increases significantly when the annulus is in motion.
\end{abstract}

Keywords- Miscible displacement flows; Non-Newtonian; Moving geometry; Annulus

\section{INTRODUCTION}

Primary cementing is a key operation in the construction of oil\&gas wells. This operation is executed in oil\&gas wells for a number of reasons, such as providing a mechanical support for the well, preventing water penetration into the well, protecting the casing from corrosive materials, to name a few. Failure in primary cementing may results in a decrease in production [1] and/or environmental consequences.

Removal of drilling mud from the annulus and its replacement with cement slurry is the foundation of the primary cementing process. The efficiency of the process depends on how successfully cement is able to displace mud out the flow geometry (i.e., an annulus). Therefore, to improve the process, displacement flows need to be studied [2].

There is currently no robust method to fully and completely displace the drilling mud in the casing and more importantly in the annulus section of the well. Recently, in the industrial setting it is becoming more common to use certain movements of the casing to improve the displacement. The current casing movements are slow reciprocation and/or rotation, aiming to increase the displacement efficiency. There exist various types of casing heads and special adaptors which make the rotation and/or reciprocation of the casing possible. Typical casing reciprocations are between 6-12 $\mathrm{m}$ over a few minutes and casing (or liner) rotations vary between 10-40 rpm. Although the precise effects of these movements are not known, they are thought to be able to break the gel strength of the drilling mud to finally mobilize the slowly moving and static mud in the annulus $[3,4,5]$.

Displacement flows of primary cementing are hard to fully understand due to the presence of various parameters. For example, the parameters that describe the geometry (inclined pipe and annulus), the inclination angle, gravitational acceleration and the flow rate require at least eight dimensionless groups to describe the flow. Considering twofluid displacement flows, this increases to 10-12 dimensionless groups [2]. Rheological properties of the fluids involved also further contribute to the complexity of the system [6,7]. To date, several studies have been conducted on different aspects of displacement flows to gain insights crucial understanding. Miscible displacement flows of Newtonian fluids in uniform pipes have been studied in detail in an inclined pipe [8, 9], a stationary vertical pipe [10] and a moving pipe [11]. Displacement flows of non-Newtonian fluids have also been studied experimentally and numerically [12, 13, 14]. Buoyant miscible displacement flows in nearly-horizontal, inclined and vertical ducts have also received attention [15, 16, 17]. Annular displacement flows have been investigated in only a few studies $[18,19]$. There even fewer studies in literature which can be directly relevant to the displacement flows in moving geometries $[7,20]$. To fill an obvious lack in the literature, in this novel work, we experimentally investigate the displacement of a viscoplastic fluid in a moving annulus. In addition to providing essential scientific knowledge, this work is also technically important as it considers for the first displacement flows in a moving annulus with moving parameters similar to those used in industrial settings.

\section{EXPERIMENTAL SETUP, PROCEDURES AND DETAILS}

Our experiments were performed in a $270 \mathrm{~cm}$ long annulus. The inner cylinder had an outer diameter of $40 \mathrm{~mm}$ and the outer cylinder had a diameter of $50 \mathrm{~mm}$, creating a gap of 5 $\mathrm{mm}$ between two cylinders. The cylinders were transparent 
(plexiglass clear acrylic cylinders) and they positioned vertically. They system had a manual plastic socket-weld gradual On/Off gate valve located at $40 \mathrm{~cm}$ from the top end (see figure 1). The gate valve initially separated the two parts of the annulus setup and completely segregated the displacing and displaced fluids. The gate valve system was designed in a way that it could be manually opened at the beginning of each experiment. All the experiments were performed at room temperature. The displacement flow setup was held on a fixed platform and it was divided into two parts: fixed and mobile sections, which enabled us to investigate the displacement flow in a stationary and moving geometry. The fixed part of the annulus setup included the outer cylinder, the clear cube vessel and all connections above the gate valve. The mobile section contained a stepper motor connected to the inner cylinder to produce the reciprocation motion. The maximum amplitude and the maximum frequency of reciprocation motion could be $15 \mathrm{~cm}$ and $0.3 \mathrm{~Hz}$, respectively. The LabVIEW was used to control all parameters. Initially, the lower part of the annulus was filled using a peristaltic pump with a Carbopol solution (light fluid or displaced fluid) colored with a small amount of ink (Fountain Pen India black ink) for visualization purposes. Light absorption calibrations were performed in usual fashion, for which the amount of $2 \mathrm{~g}$ of ink per one liter of liquid was found to be a reasonable trade-off. The upper part of the annulus, above the gate valve, was filled with displacing fluid (heavy fluid). The densities of our experimental fluids were measured by a high-precision density meter (Anton Paar DMA 35 ). The viscosity and the yield stress of the solutions were measured in usual fashion, using AR-G2 TA Instrument digital controlled shear stress-shear rate rheometer. The maximum error in measurement of viscosity was $5 \%$. To avoid pump disturbances and ensure a smooth steady inflow, the displacing upper fluid was fed by gravity from a large elevated tank filled with the displacing fluid. The flow rate was controlled by a needle valve and it was measured by a flow meter (Omega FTB 421, low flow plastic turbine), located downstream of the annulus setup. At the start of each experiment, the gate valve was opened and the displacement process started. Images of the displacement flow were recorded using a high-speed camera (Basler acA2040, with 4096 gray-scale levels) covering around $120 \mathrm{~cm}$ below the gate valve and they were subsequently postprocessed using in-house Matlab codes, to quantify different aspects of the flow. To enhance quality of the images, the annulus setup was back-lit using Light-Emitting Diode (LED) strips. In addition, to improve light homogeneity, a diffusive layer was placed between the annulus setup and the LED strips.

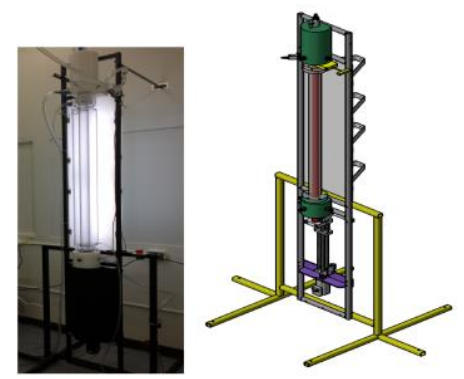

Figure1. Real and schematic views of experimental annulus setup.

\section{RESUlTS AND DisCUSIONS}

The experiments were performed at a fixed density difference and at a small Atwood number $\left(A t=\frac{\hat{\rho}_{H}-\hat{\rho}_{L}}{\hat{\rho}_{H}+\hat{\rho}_{L}}=0.0005\right) \cdot \hat{\rho}_{H}$ and $\hat{\rho}_{\mathrm{L}}$ represent the densities of the displacing and displaced fluids, respectively. The displaced fluid was a Carbopol solution as a viscoplastic fluid with a $1.4 \mathrm{~Pa}$ yield stress. The displacing fluid was a Xanthan solution. Carbopol and Xanthan compositions and determined parameters from rheological measurements, assuming the Herschel-Bulkley model (Eq. 1), and power low model (Eq. 2) are listed in Table.1, respectively.

$$
\begin{array}{r}
\hat{\tau}=\hat{\tau}_{Y}+\hat{\kappa} \hat{\gamma}^{\mathrm{n}} . \\
\hat{\tau}=\hat{\kappa} \hat{\gamma}^{\mathrm{n}} .
\end{array}
$$

TABLE I. RHEOLOGICAL MEASURMENTS FOR CARBOPOL \& XANTHAN

\begin{tabular}{|c|c|c|c|}
\hline \multicolumn{4}{|c|}{ Rheological measurements for Carbopol composition } \\
\hline & Consistency & $\begin{array}{c}\text { Power-law } \\
\text { index }\end{array}$ & $\begin{array}{c}\text { Yield } \\
\text { stress }\end{array}$ \\
\cline { 2 - 4 } & $\hat{\kappa}\left(\mathrm{Pa} \mathrm{s}^{\mathrm{n}}\right)$ & $\mathbf{n}$ & $\hat{\tau}_{\mathrm{Y}}(\mathrm{Pa})$ \\
\hline Carbopol $0.07 \%(\mathrm{wt} / \mathrm{wt})$ & 1.34 & 0.45 & 1.4 \\
\hline Xanthan $0.25 \%(\mathrm{wt} / \mathrm{wt})$ & 0.81 & 0.31 & ---- \\
\hline
\end{tabular}

The Herschel-Bulkley and power low models are appropriate rheological models that describes the shear behavior of the Carbopol gel and Xanthan solutions, respectively. Figure 2 shows an example of the rheometer flow curve for our Carbopol solution, in which the Herschel-Bulkley model is fitted to the datapoint.

To study the effect of the reciprocation motion on the displacement flow, the annulus setup was used to impose a reciprocation motion of $100 \mathrm{~mm}$ at a small frequency $(0.1 \mathrm{~Hz})$.

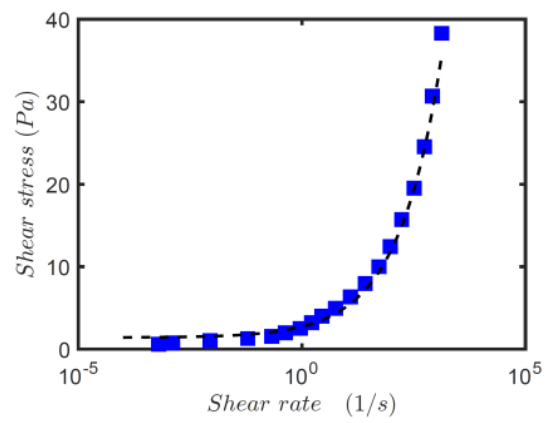

Figure 2. Rheological results for low concentration of the Carbopol solution. The dashed line indicates the Herschel-Bulkley model parameters fitted to the data. 


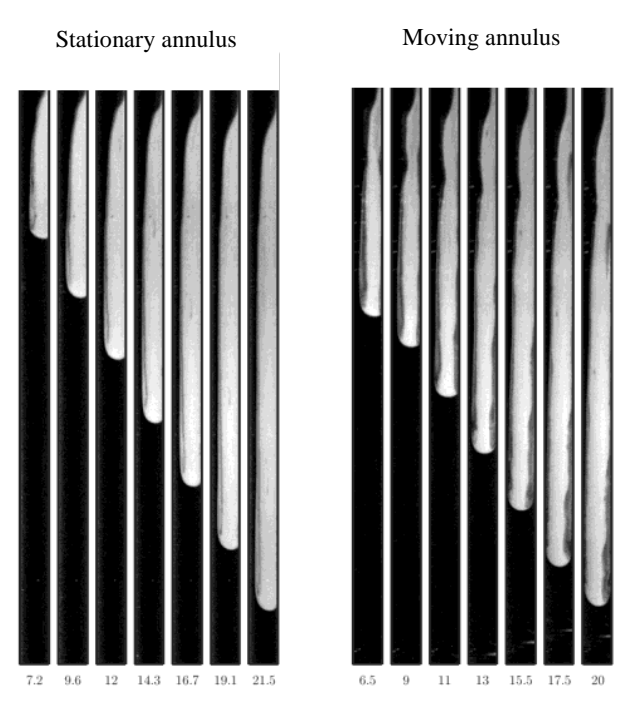

Figure 3. Sequence of images showing the displacement flow in a stationary and moving geometry (with a frequency of $0.1 \mathrm{~Hz}$ over $100 \mathrm{~mm}$ amplitude with respect to vertical). The field of view is $46 \times 1200 \mathrm{~mm}^{2}$ right below the gate valve. From left to right, the results of stationary and moving geometry are shown at $\hat{\mathrm{V}}_{0} \approx 14(\mathrm{~mm} / \mathrm{s})$. The experimental times in each image sequence panel are provided in second (s), below each snapshot.

Figure 3 shows examples of our annular displacement flow results. This figure (left side) shows a sequence of images of a flow in stationary annulus at a low imposed flow rate, i.e., $\hat{\mathrm{V}}_{0}=13.74(\mathrm{~mm} / \mathrm{s})$. As time grows, the heavy fluid displaces the light fluid in the downward direction and there is not much mixing between the two fluids. In addition, there is a static residual layer of the Carbopol solution on the wall. The same behavior is observed in figure 3 (right side) and it shows that the displacement process is not as efficient as the case without motion at short time. However, it should be mentioned that the story is different at extended times.

Figure 4 shows a sequence of experimental images with the same parameters of figure 3 but for an extended time. This figure indicates the effects of a moving geometry on the flow at longer times. It is obvious that the static residual layer is evidently stable and the amount of the Carbopol solution remains unchanged for the stationary geometry (figure 4). Figure 4 (right side) shows that by adding a reciprocation motion, the overall displacement improves: the amount of the Carbopol displaced by Xanthan solution is increased. In fact, the reciprocating motion of the inner cylinder is able to remove the static residual layers of the displaced phase, in the downward direction. Due to the shear exerted by the inner wall motion, the reciprocation enhances the removal of Carbopol from the walls and consequently the displacement flow efficiency increases.
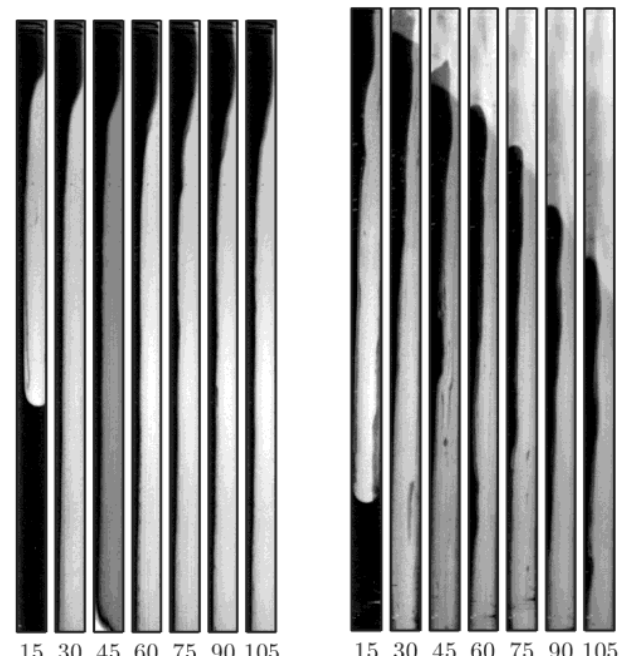

Figure 4. Sequence of images showing the displacement flow in a stationary and moving geometry (with a frequency of $0.1 \mathrm{~Hz}$ over $100 \mathrm{~mm}$ amplitude with respect to vertical) at long time. The field of view is $46 \times 1200 \mathrm{~mm}^{2}$ right below the gate valve. From left to right, the results of stationary and moving geometry are shown at $\hat{\mathrm{V}}_{0} \approx 14(\mathrm{~mm} / \mathrm{s})$. The experimental times in each image sequence panel are provided in second (s), below each snapshot.

To explain this feature more quantitatively, it is necessary to introduce the depth-averaged concentration profiles of our displacements (shown by $\mathrm{C}$ in the following figures). Figure 5 shows an example of the evolution of the average concentration profile with time and streamwise location. Note that the experimental snapshots are calibrated between 0 (dark displaced fluid) and 1 (transparent displacing fluid). Therefore, in these diagrams, when the concentration value is around 1 , the displacement is complete and there is no layer of the displaced fluid on the wall. On the other hand, when the concentration value is smaller than 1 a layer of the Carbopol solution remains on the wall.

There are interesting results delivered through analyzing depth-averaged concentration diagrams. First, the depthaveraged concentration profiles show the displacement flow is not complete and a layer of the displaced fluid remains on the wall at all times. Second at short times, the motion does not seem to affect the displacement efficiency. 

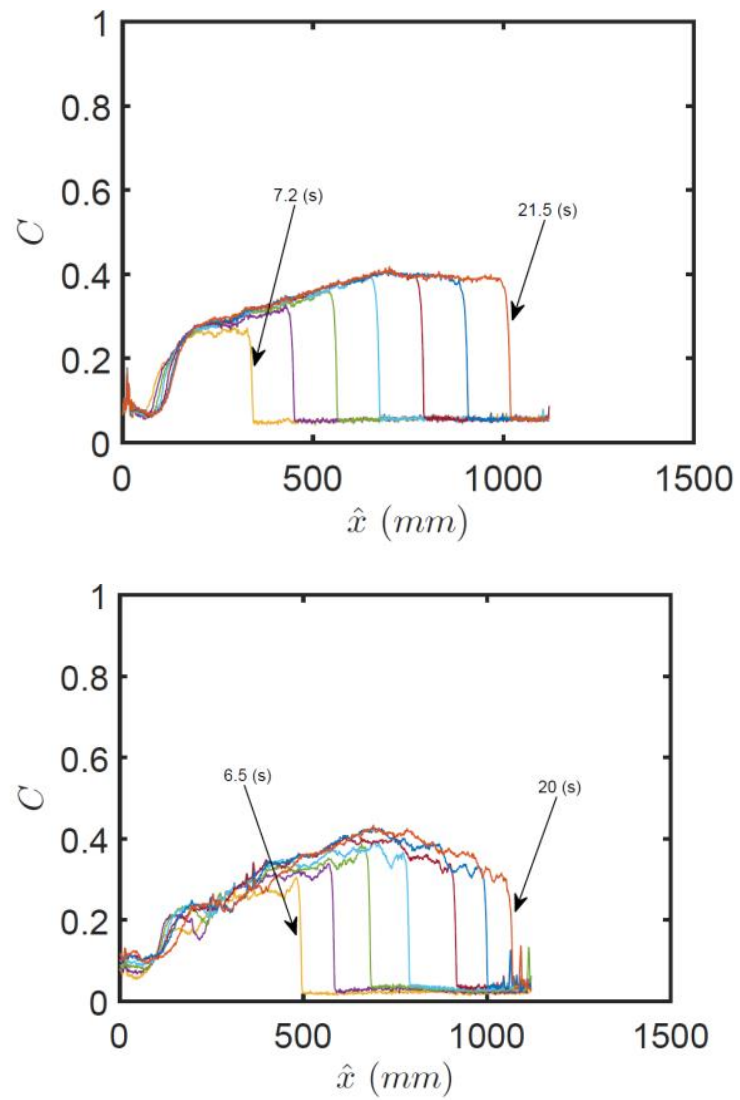

Figure 5. Evolution of the depth-averaged concentration field with time $\hat{\mathrm{t}}$, and streamwise location, $\hat{x}$ measured from the gate valve. From top to bottom, the geometry is stationary $\quad(\hat{\mathrm{t}}=[7.2,9.6,12,14.3,16.7,19.1,21.5](\mathrm{s})) \quad$ and moving $(\hat{\mathrm{t}}=[6.5,9,11,13,15.5,17.5,20](\mathrm{s}))$, respectively.

To give a global vision of the displacement efficiency at longer times, the depth-averaged concentration diagrams with the same parameters are presented in figure 6. It can be seen that when the Carbopol solution is displaced by the Xanthan solution in the moving geometry, the amount of Carbopol layer on the wall decreases, significantly. In the stationary geometry of figure 6 (top), the concentration values are approximately below 0.5 and all profiles nearly collapse. Therefore, as time grows, the efficiency of the displacement flow does not change. However, in the moving geometry of figure 6 (bottom) the concentration value is under 0.8 . Therefore, the amount of the Carbopol solution on the wall decreases significantly and the displacement efficiency increases at an extended time.
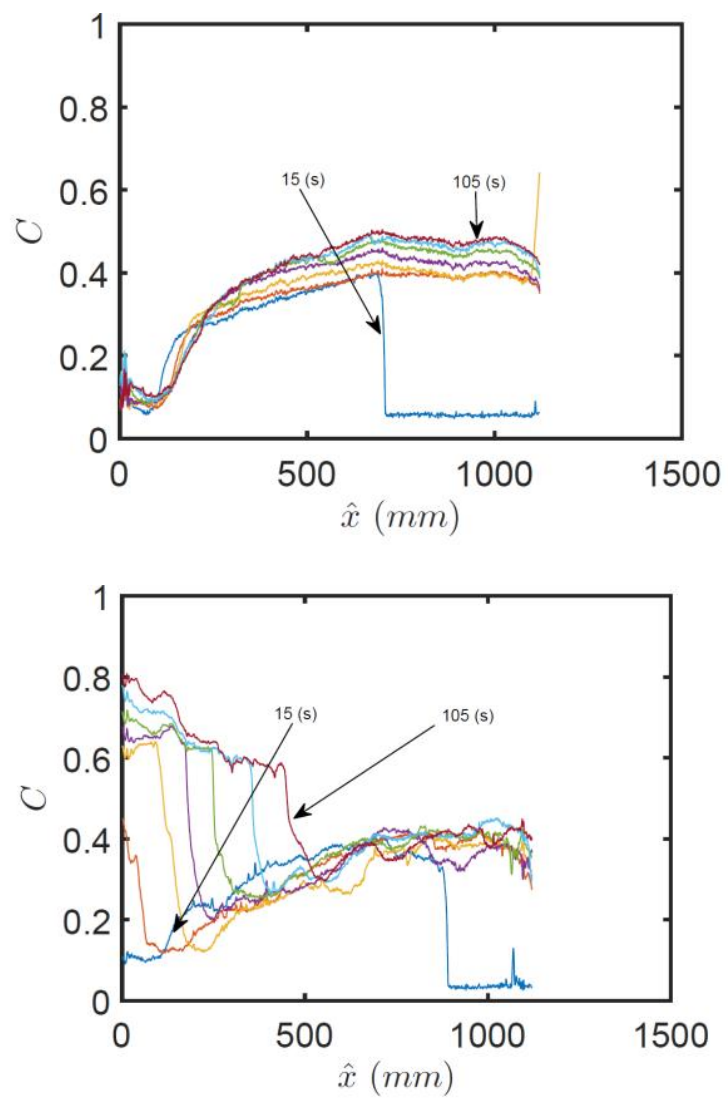

Figure 6. Evolution of the depth-averaged concentration field with time $\hat{\mathrm{t}}=[15,30,45,60,75,90,105](\mathrm{s})$, and streamwise location, $\hat{\mathrm{x}}$ measured from the gate valve. From top to bottom, the geometry is stationary and moving, respectively.

\section{CONCLUSION}

We experimentally studied miscible displacement flows in a long, vertical moving annulus. The experimental fluids were Xanthan solution (displacing fluid) and non-Newtonian viscoplastic (displaced fluid) and they had a very small density difference. The main finding was that the displacement flow efficiency was a function of the geometry motion. In the cases studied, the displacement flow in the moving annulus (reciprocation motion) was more efficient in comparison to the stationary annulus.

\section{RENFERNCES}

[1] G. D. Nelson EB, Well cementing, 2nd edn (2006).

[2] I. A. Frigaard, K. G. Paso, P. de Souza Mendes, Binghams model in the oil and gas industry, Rheologica Acta 56 (3) (2017) 259-282.

[3] N. Moroni, K. Ravi, T. Hemphill, P. Sairam, et al., Pipe rotation improves hole cleaning and cement-slurry placement: Mathematical modeling and field validation, in: Offshore Europe, Society of Petroleum Engineers, 2009.

[4] M. Carrasco-Teja, I. Frigaard, Displacement flows in horizontal, narrow, eccentric annuli with a moving inner cylinder, Physics of Fluids 21 (7) (2009) 073102. 
[5] M. Carrasco-Teja, I. Frigaard, Non-newtonian fluid displacements in horizontal narrow eccentric annuli: effects of slow motion of the inner cylinder, Journal of Fluid Mechanics 653 (2010) 137-173.

[6] R. C. Haut, R. J. Crook, et al., Primary cementing: The mud displacement process, in: SPE Annual Technical Conference and Exhibition, Society of Petroleum Engineers, 1979.

[7] C. Holt, N. Lahoti, V. Fortier, New cementing method uses pipe movement to maximize displacement (2013).

[8] K. Alba, S. Taghavi, I. Frigaard, Miscible density-stable displacement flows in inclined tube, Phys. Fluids 24 (2012) 123102.

[9] T. Seon, J. Znaien, D. Salin, J. Hulin, E. Hinch, B. Perrin, Front dynamics and macroscopic diffusion in buoyant mixing in a tilted tube, Phys. Fluids 19 (2007) 125105.

[10] A. Amiri, F. Larachi, S. Taghavi, Buoyant miscible displacement flows in vertical pipe, Phys. Fluids 28 (10) (2016) 102105.

[11] A. Amiri, F. Larachi, S. Taghavi, Displacement flows in periodically moving pipe: understanding multiphase flows hosted in oscillating geometry, Chemical Engineering Science.

[12] M. Ben Amar, E. Poire, Pushing a non-newtonian fluid in a hele-shaw cell: From fingers to needles, Phys.Fluids 11 (7) (1999) 1757-1767.

[13] I. Frigaard, D. Ryan, Flow of a visco-plastic fluid in a channel of slowly varying width, J.non-Newt.Fluid Mech. 123 (1) (2004) 67-83.

[14] S. Taghavi, K. Alba, M. Moyers-Gonzalez, I. Frigaard, Incomplete fluidfluid displacement of yield stress fluids in nearhorizontal pipes: experiments and theory, J.non-Newt.Fluid Mech. 167-168 (2012) 59-74.

[15] K. Alba, S. Taghavi, I. Frigaard, Miscible density-unstable displacement flows in inclined tube, Physics of Fluids 25 (6) (2013) 067101.

[16] S. Taghavi, T. Seon, D. Martinez, I. Frigaard, Influence of an imposed flow on the stability of a gravity current in a near horizontal duct., Phys. Fluids 22 (2010) 031702.

[17] R. Mollaabbasi, S. Taghavi, Buoyant displacement flows in slightly nonuniform channels, J. Fluid. Mech 795 (2016) 876-913.

[18] M. Tehrani, S. Bittleston, P. Long, Flow instabilities during annular displacement of one non-newtonian fluid by another, Experiments in Fluids 14 (4) (1993) 246-256.

[19] S. Pelipenko, I. Frigaard, Two-dimensional computational simulation of eccentric annular cementing displacements, J. Engng. Math. 64 (6) (2004) $557-583$.

[20] M. Carrasco-Teja, I. Frigaard, B. Seymour, S. Storey, Visco-plastic fluid displacements in horizontal narrow eccentric annuli: stratification and traveling wave solutions, J. Fluid Mech. 605 (2008) 293-327. 